\title{
The instability strip and pulsation features of post-AGB star models
}

\author{
T. Aikawa
}

Tohoku gakuin University, Sendai 981-3193, Japan

e-mail: aikawa@cs.tohoku-gakuin.ac.jp

Received 27 July 2009 / Accepted 15 January 2010

ABSTRACT

\begin{abstract}
Aims. The instability strip and characteristics of the nonlinear behavior of the radial pulsation are examined for the observed photometric variability of post-AGB stars.

Methods. A linear stability analysis for radial pulsation modes is performed in the domain $3.70 \leq \log T_{\text {eff }} \leq 3.90,0.0 \leq \log g \leq 1.8$, assuming a total mass $0.6 M_{\odot}$ and $0.8 M_{\odot}$ for post-AGB stars. Radial modes up to the 5th overtone are considered, since we expect that there are some strange modes amongst high-order modes. Nonlinear simulations are tried for all the models, which have at least one pulsationally unstable mode.

Results. Pulsationally unstable modes are found in a large region of the domain, and these modes may be responsible for the observed variability. Nonlinear simulations on models that have unstable modes with higher overtones reveal the variability through small peakto-peak variations. These variations are characteristics of the observed variations for some post-AGB stars located in the bluer region of the classical instability strip.
\end{abstract}

Key words. stars: oscillations - stars: AGB and post-AGB - hydrodynamics

\section{Introduction}

Post-AGB stars are luminous objects that evolve on a fast track to hotter effective temperatures at roughly constant luminosity after AGB evolution by a very strong mass loss. In the course of such evolution they cross the classical instability strip extended to higher luminosity. Photometric variability observed in some post-AGB stars thus are believed to be caused by stellar pulsation. However, the behavior of pulsation in the post-AGB stars may be quite different from those in classical Cepheid variables, since the post-AGB stars are low mass stars but they have high luminosities.

In this study, we first summarize the behavior of the photometric variability for a selected sample of post-AGB stars and post-AGB candidates. Then we construct the envelope models for linear analysis and nonlinear simulation of radial pulsation, intending to explain the observed photometric variability due to radial pulsation of post-AGB envelopes.

Pulsation is sometimes a key to understanding the nature of post-AGB stars (Córsico et al. 2007) and post-AGB related stars, such as FG Sagittae (Whitney 1978; Jeffery \& Schönberner 2006). For a specific star, HD 56126, Jeannin et al. (1996, 1997) and Barthès et al. (2000) reported the results of nonlinear simulation of radial pulsation for comparison with the observed light variation. This study was the first attempt at direct comparison of the variability in post-AGB stars with theoretical nonlinear pulsation models.

Kiss et al. (2007) studied the instability strip of post-AGB stars based on 30 known and candidate binary post-AGB stars. They confirmed the instability strip; besides this, on the bluer side of the instability strip, they found some variable stars where the peak-to-peak light-curve amplitudes are relatively small (less than $0.5 \mathrm{mag}$ ) compared with the major stars in the strip. They found a few similar stars at redder side, too.

Schmidt (2001) presents a table of spectroscopic parameters $\left(T_{\text {eff }}\right.$ and $g$ ) of selected post-AGB stars based on the literature. We start our discussion with post-AGB stars in the table to confirm the existence of the region for the small amplitude variables that are bluer than the classical instability strip, as mentioned by Kiss et al. (2007) and to define the extension of the region of small-amplitude variables beyond the classical instability strip, though this table is preliminary (cf. Szczerba et al. 2007). We first searched in the literature for the photometric variability of the post-AGB stars in the table. We summarize the result in Fig. 1. It is pointed out that the photometric variability in the literatures is mainly reported for the stars in the late type region of the HR diagram, and variable stars with small amplitude (the peak-to-peak variation less than 0.5 ) are located in a hotter part of the region. Pulsation stars classified as RV Tau stars are located in the main part of the region.

In summary, the stars that show photometric variability are confined to the late type region where $\log T_{\text {eff }}$ is less than 3.90. The small-amplitude variations are observed in a bluer region of the main domain. In Fig. 1, in the small-amplitude region, there are several stars (i.e., IRAS Z02229+6208, IRAS 04296+3429, ROA 24, AFGL 2688) that have no remarks on the variability and that might also have small-amplitude variations.

Probably in the table we have missed some interesting variable stars in the early-type region classified as luminous hothelium stars (Saio and Jeffery 1988). The cause of variability is known as iron-group bump in the opacity (Saio 1995; Fadeyev \& Lynas-Gray 1996), and the stars are definitely related to postAGB stars.

In this paper, we assume that the variability of the postAGB stars is caused by radial pulsation of the envelope. In 


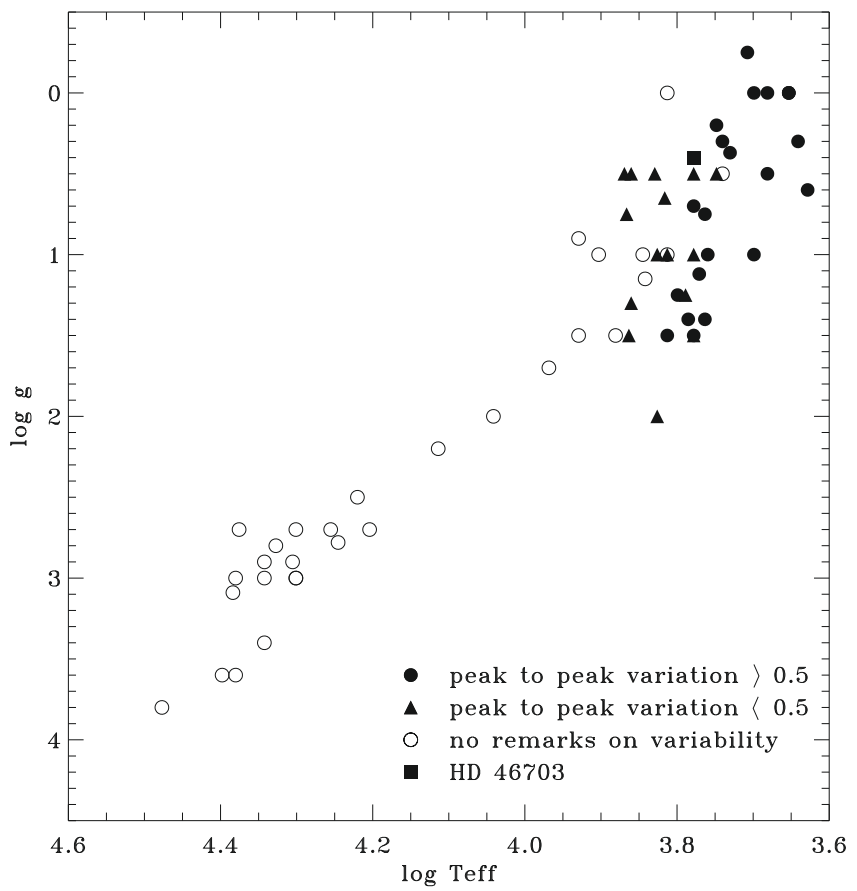

Fig. 1. The features of photometric variability of post-AGB on the $\log g \log T_{\text {eff }}$. Filled circles indicate stars with peak-to-peak variation greater than $0.5 \mathrm{mag}$, empty circles correspond to stars with no remarks on photometric variability, and filled triangles depict variable stars of the peak-to-peak variation less than 0.5. HD 46703 (see text) is marked with a filled square.

particular, we stress that the small-amplitude variability may be explained by the strange mode in radial pulsation. For examining the amplitude of model pulsation, we perform nonlinear simulation to get the pulsation behavior in the nonlinear regime. We first examine the linear stability of radial modes, and then the nonlinear simulations are performed for the models with pulsationally unstable modes. The nonlinear simulation, as well as linear analysis, are performed with a hydrodynamic code with the variable Eddington factor approximation of radiative transfer (Aikawa 2008).

In this paper we have entirely ignored convection effects. For a static point of view, convection acts an energy transporter, so the radiative transfer becomes less effective than a purelly radiative envelope. It is expected that convection may stabilize pulsation. But there must be a dynamic coupling between convection and pulsation. It is thus desirable to include convection, although Stothers (2003) studies the effects of convection on the stability of luminous envelopes qualitatively.

\section{Hydrostatic models}

We first construct the envelope models according to observed effective temperature $\left(\log T_{\text {eff }}\right)$ and surface gravity $(\log g)$. We adopt 0.6 and $0.8 M_{\odot}$ as the total mass of post-AGB stars. According to the hydrogen-rich envelope mass vs. the effective temperature relationship by Paczyǹski (1971) and Schönberner (1981, 1983), Blöcker (1995) and Frankowski (2003), the envelope mass in the range of observed effective temperature is about $1 / 100 M_{\odot}$, so the total mass should be equal to the core mass. To convert $\log g$ to the luminosity, we use the following equation with using $T_{\text {eff }}$ and $\log g$ are $5777 \mathrm{~K}$ and 4.4378 for the Sun, respectively (Allen 2000):

$\log \left(L / L_{\odot}\right)=4 \log T_{\text {eff }}+\log \left(M / M_{\odot}\right)-\log g-10.6091$.
The hydrostatic equilibrium envelope models were constructed as described by Aikawa (2008). In this method the variable Eddington factor approximation is applied to radiative hydrodynamics instead of the diffusion approximation. Chemical abundances are assumed as $X=0.700, Y=0.298, Z=0.002$. The metal abundance ratio is a simplified version of the solar abundance ratio. We used OP opacity (Seaton 1992; Seaton et al. 1994) for the Roseland mean and Planck mean opacities. For regions of low density and low temperature, the opacities supplied by Alexander \& Ferguson (1994) are used. The equations of radiative hydrodynamics are reduced to finite difference equations, and there are about 200 zones for the difference equations. Equations of the hydrostatic equilibrium and equation of radiative transfer are solved iteratively until obtaining a consistent solution. We denote the Eddington factor in the hydrostatic equilibrium as $f_{\text {eq }}$. The hydrostatic equations are integrated inward from the surface, defined as the radius of the optical depth $\tau=0.001$, until the ratio of the radius $(R)$ of the bottom to one at the surface $\left(R_{\mathrm{S}}\right)$ becomes less than 0.05 . For the mass $\left(1-M / M_{\mathrm{S}}\right)$, it is 0.005 at most. We assume that radial pulsation is confined to the region defined by the two radii.

\section{Linear analysis}

The linear nonadiabatic eigenvalue problem for radial modes was solved by using $f_{\text {eq }}$ as the Eddington factor. While sometimes the Eddington factor estimated by the linear perturbed quantities may yield quantitatively different results on the growth rates for strange modes (Zalewski 1992), we used $f_{\text {eq }}$ as the Eddington factor for linear analysis for simplicity. Since we are interested in the strange mode in post-AGB models, higher modes up to the fifth overtone were examined.

Since the present models have strong nonadiabatic effects, the periods and the growth rates deviate strongly from those in the adiabatic approximation (Wood 1976). We thus followed Aikawa (2008) to get the periods and the growth rates of radial pulsation at the fully nonadiabatic condition. Starting in the adiabatic condition, the periods and the growth rates were calculated step-by-step by increasing the effects of nonadiabaticity. The increment of the effect was carefully set to ensure the succession in the order of modes from those of an adiabatic case where we may know the complete set of the eigen-value problem. Thus, we may label the order of modes in fully nonadiabatic approximation with the correspondig modes in adiabatic condition, and so sometimes the order of modes in the fully nonadiabatic approximation is not the decrease in the periods, which is a definition of the order of modes in adiabatic condition(see, Fig. 2). The periods of strange modes that appear as unstable high-order modes with high growth rates seriously drift among those of other modes. Probably this comes from the strange modes being caused mainly by the hydrogen ionization zone at outer envelopes (Aikawa \& Sreenivasan 1996), so those modes are strongly affected by nonadiabatic effects.

The results are summarized in Fig. 2 for $0.6 M_{\odot}$. For lower effective temperature models, such as $\log T_{\text {eff }}=3.70$, the fundamental and/or the 1st-overtone modes are pulsationally unstable. For higher effective temperature models, however, these loworder modes become stable but high-order modes become unstable. This tendency first appears in the models of middle $\log g$ values. Finally, all models are stable in the low-order modes, but unstable in high-order modes at higher effective temperature (for instance, $\log T_{\text {eff }}=3.85$ ). For $\log T_{\text {eff }}=3.90$, all the modes up to the 5 th overtone are stable in the range of $\log g$ relevant to the observed post-AGB. The results are summarized in Fig. 3 for 
T. Aikawa: The instability strip and pulsation features of post-AGB star models
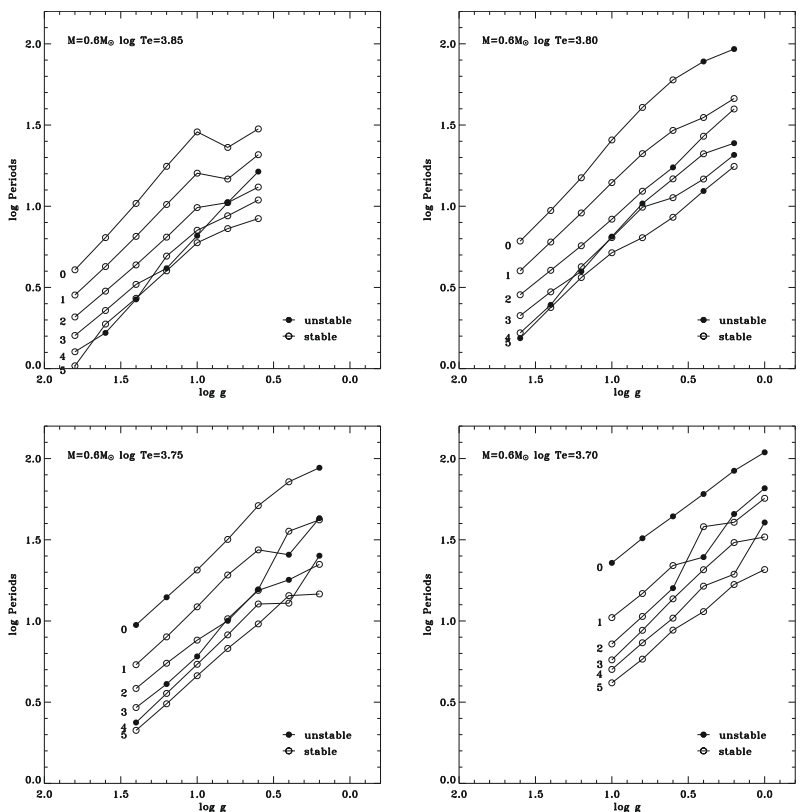

Fig. 2. The results of linear analysis for model sequences for fixed effective temperatures $\left(\log T_{\text {eff }}=3.85,3.80,3.75,3.70\right)$ for $M=0.6 M_{\odot}$. The ordinate corresponds to $\log g$ of the models, and the abscissa corresponds to the linear pulsation periods in a logarithmic scale. Linear nonadiabatic analysis is applied to radial modes up to the 5th overtone mode. The numbers at left of each curve denote the radial order of the modes ( 0 : the fundamental, 1 : the 1 st overtone ...). Pulsationally stable modes are indicated by a hollow circle, and unstable modes are marked by a filled circle.
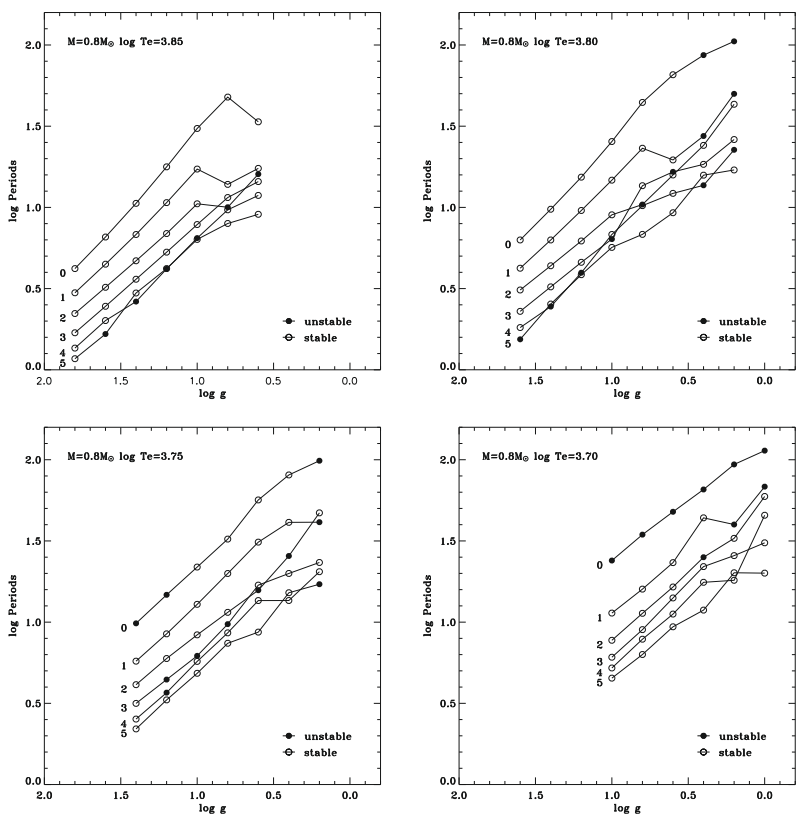

Fig. 3. Same as Fig. 2, but for the case of $M=0.8 M_{\odot}$.

$0.8 M_{\odot}$. The characteristics of instability are almost the same as in the case of $0.6 M_{\odot}$. For $\log T_{\text {eff }}=3.90$, all the modes are also stable.

For both the masses of $0.6 M_{\odot}$ and $0.8 M_{\odot}$, there are pulsationally unstable modes of high overtone in the model sequences of higher effective temperatures $\left(\log T_{\text {eff }}=3.85,3.80,3.75\right)$. Aikawa $(1991,1993)$ showed that these pulsationally unstable high-order modes have the characteristics of the strange mode.

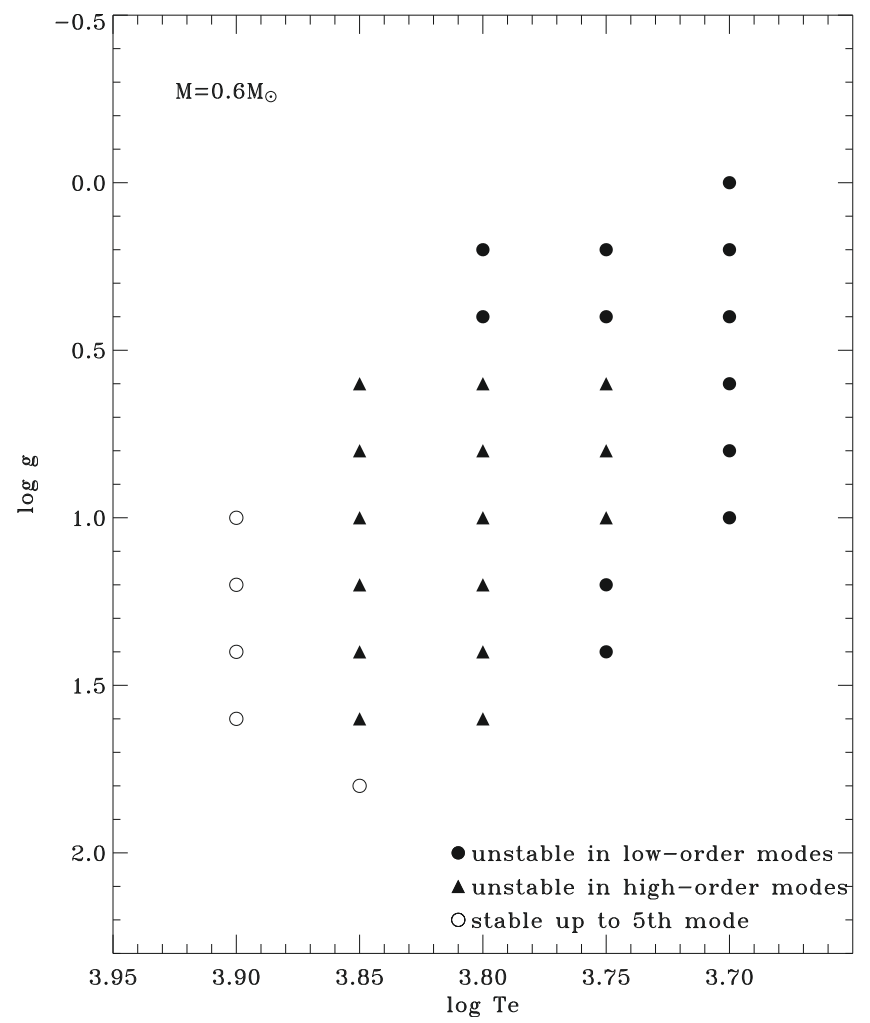

Fig. 4. The instability strip resulting from the linear analysis for $M=$ $0.6 M_{\odot}$. Models that are pulsationally stable up to the 5th overtone modes are shown by circles, models where the fundamental and/or the 1st-overtone modes are unstable are displayed with filled circles, and models where only higher modes than 1st-overtone mode are unstable are depicted with filled triangles.

In some models, only these high overtone modes are unstable, so these strange modes should be responsible for the photometric variability, if radial modes are assumed to be the cause of that.

In Figs. 4 and 5, we show summary maps of the instability strip of the radial modes for $M=0.6 M_{\odot}$ and $M=0.8 M_{\odot}$. For both the masses, there is a instability region that comes from high overtone modes (probably strange modes) at a hotter regime than in the case of the fundamental and 1st-overtone modes.

The instability of the fundamental, and the 1st-overtone at higher $\log g$ at $\log T_{\text {eff }}=3.75$ and 3.70 comes from the model location just inside the blue edge of the classical instability strip extended to the high luminosity of low-mass stars (for instance W Verginis stars, Bridger 1985). On the other hand, the instability due to the fundamental and the 1st-overtone modes at lower $\log g$ are those found by Saio et al. (1984) and may be related to the opacity-modified Eddington limit (Asplund 1998).

\section{Nonlinear simulation}

Nonlinear simulations for models with pulsationally unstable modes were performed with the variable Eddington factor, $f_{\text {dyn }}$ (Aikawa 2008), snapshot values of the Eddington factor at each time step. The simulation of each model was started from the hydrostatic equilibrium except for the velocity perturbation. The velocity profile was the form of the eigenfunction and the amplitude of the velocity perturbation at the surface is $1 \mathrm{~km} \mathrm{~s}^{-1}$, which is quite small compared to one expected to the limit cycles of classical Cepheids. 


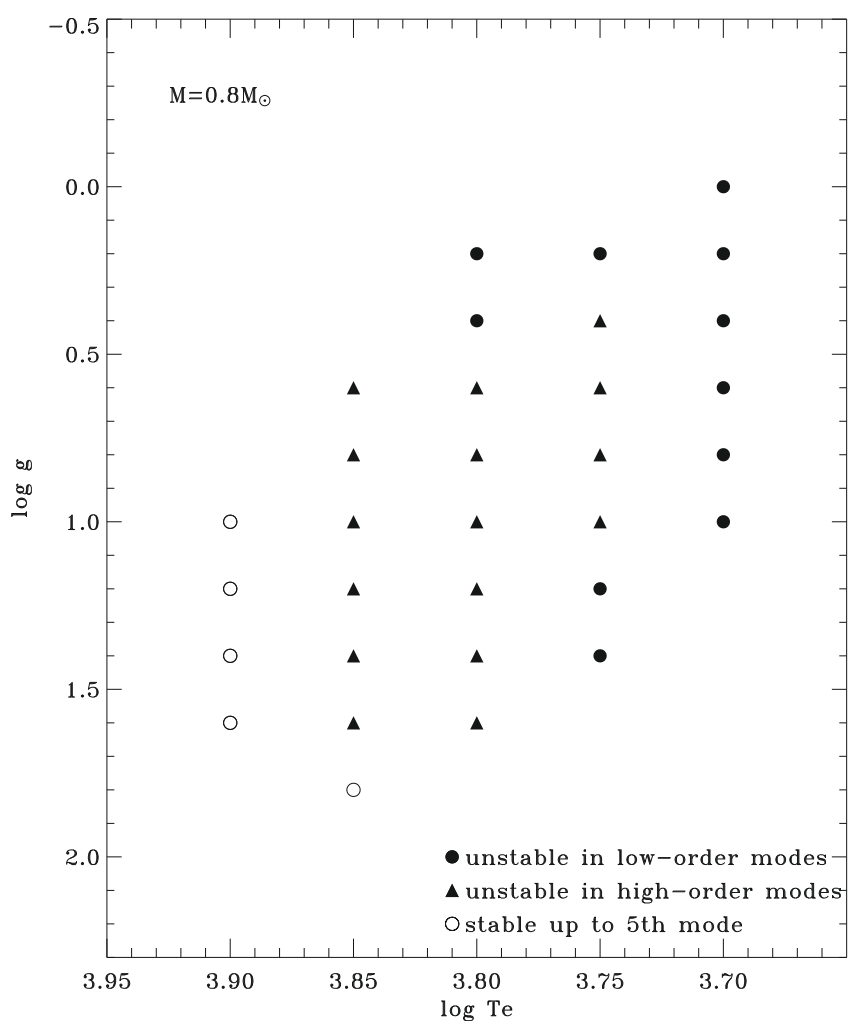

Fig. 5. Same as in Fig. 4, but for $M=0.8 M_{\odot}$.

Besides not taking convection into account, the main ambiguities of nonlinear modeling come from the artificial viscosity to stabilize calculations and the boundary condition at the surface. For the artificial viscosity, we used the formula of the von Neumann-Richtmyer type with a cutoff (Stellingwerf 1975a,b), i.e.,

$Q_{i}=C_{Q} P_{i}\left[\max \left(0, \frac{u_{i}-u_{i+1}}{\sqrt{P_{i} V_{i}}}-C_{Q \text { cut }}\right)\right]^{2}$,

where $P_{i}$ and $V_{i}$ are ordinary pressure and specific volume at the cell between $i$ and $i+1$ shells, respectively, and $u_{i}$ s are velocities of the shell at $i$. The constants of $C_{Q}=4$ and $C_{Q \text { cut }}=0.02$ are used.

We assumed the perfect reflection of the wave at the surface as one of the necessary boundary condition at the outer boundary:

$P_{\mathrm{NP}}=P_{\mathrm{rad}}$.

The pressure $P_{\mathrm{NP}}$ at the outermost cell was assumed to be equal to the radiative pressure $P_{\text {rad }}$ evaluated by other quantities at the cells. This condition would be invalid for models in which the velocities of some outer shells exceed their escape velocities, and the wave may propagate beyond the outer boundary.

The resulting finite difference equations are time dependent and solved by the fully implicit scheme (Castor et al. 1977). Simulation for each model was followed enough to confirm that the pulsation is settled into a stationary state. The results are depicted as light curves at the model photosphere in Figs. 6-8 for $M=0.6 M_{\odot}$.

We notice that the amplitudes of nonlinear pulsation in a stationary state are quite small in higher effective temperature models (for instance $\log T_{\text {eff }}=3.85$ ). These amplitudes are much small than those observed in post-AGB variables. The lower the models effective temperature, the larger the amplitudes. For

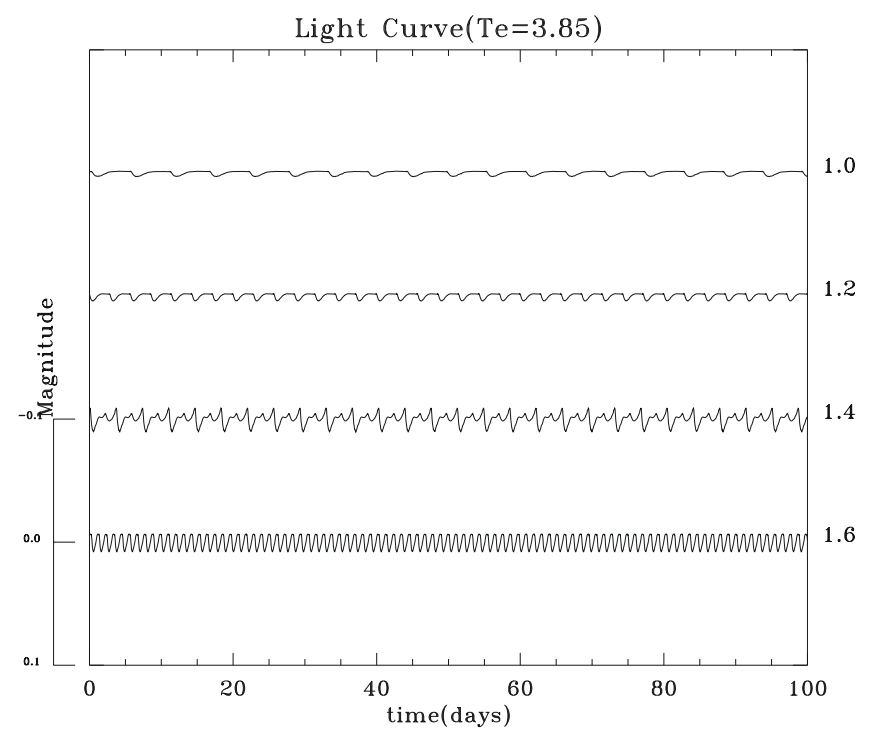

Fig. 6. The photospheric light curves predicted by nonlinear models for $\log T_{\text {eff }}=3.85$ and $M=0.6 M_{\odot}$. The ordinate corresponds to the time in unit of days, and the abscissa corresponds to the photometric magnitude. The values of the surface gravity are indicated for each curve. The scale of the magnitude is valid for each curve that is shifted vertically for clarity.

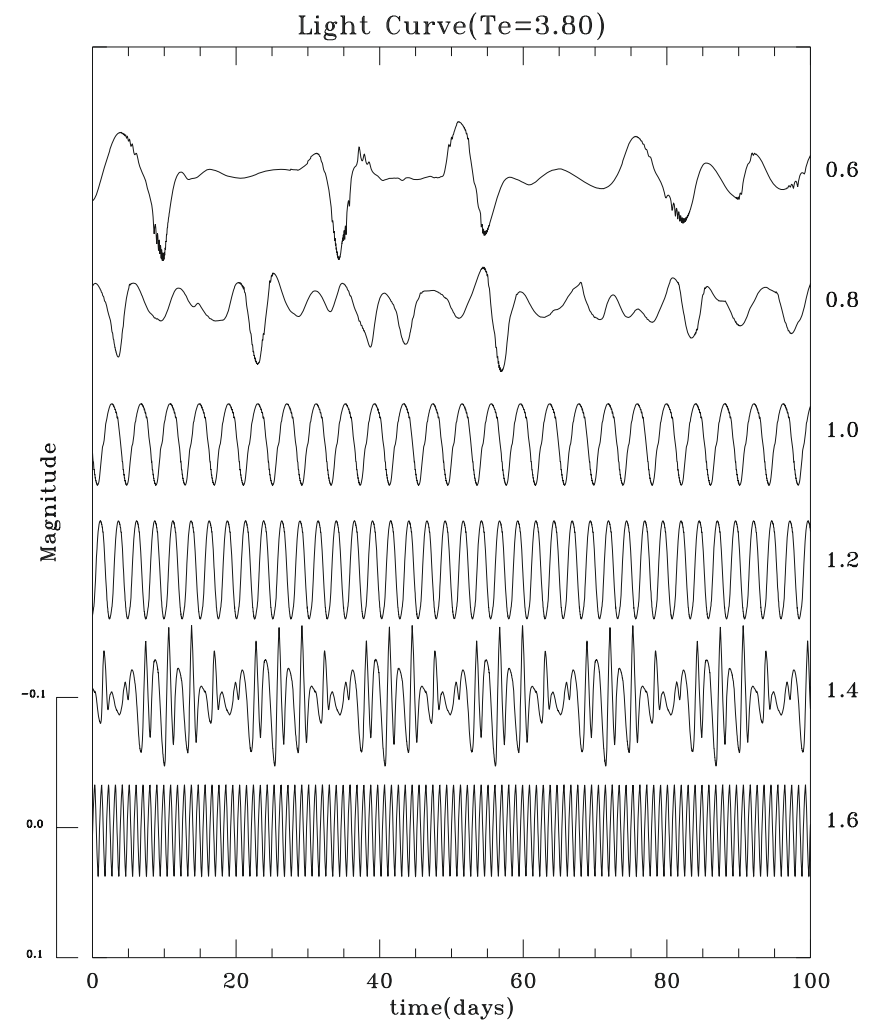

Fig. 7. Same as in Fig. 6 but for $\log T_{\text {eff }}=3.80$ and $M=0.6 M_{\odot}$.

$\log T_{\text {eff }}=3.80$, the amplitudes are comparable to those observed, though the pulsation driving modes are still high-order modes and probably strange modes. For even lower temperature, there are large amplitude pulsations at a higher $\log g$ region due to low-order modes(mainly the fundamental mode), as expected, though there are small-amplitude pulsation caused by high-order modes at lower $\log g$ region. It is known that pulsation amplitudes in stars in the instability strip of low-mass supergiant stars 


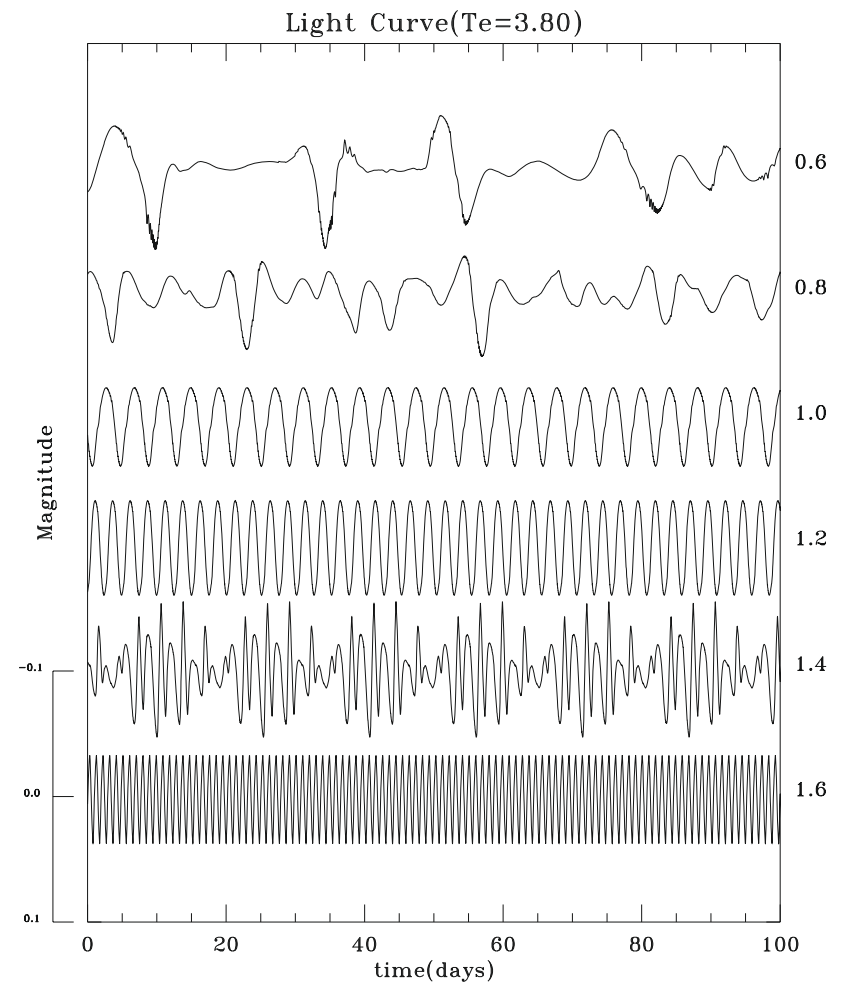

Fig. 8. Same as in Fig. 6 but for $\log T_{\text {eff }}=3.75$. The upper scale is applied to the upper four curves, and the lower scale is valid for the lower three curves. Only the transient light curve is plotted for $\log g=$ 1.2 .

classified as W Virginis stars are quite large with propagating shock wave features (Davis 1972). The fundamental pulsation at higher $\log g$ is the same. We also notice that stationary pulsations at lower $\log g$ models are irregular with small amplitudes, though those at higher $\log g$ are regular. This feature of nonlinear pulsation may be useful for comparing to observed features.

Figures 9-11 show the results for $M=0.8 M_{\odot}$. For a wide range of the HR diagram the pulsation behavior for a parameter set with $\log T_{\text {eff }}$ and $\log g$ is essentially the same as in the model of the same parameter of $M=0.6 M_{\odot}$. For $\log T_{\text {eff }}=3.85$, quite regular pulsations are realized but these are too small amplitudes again. For lower temperatures, such as $\log T_{\text {eff }}=3.80$, the amplitudes of regular pulsation become larger, and irregular pulsation is also realized for lower gravity models. For even lower temperature, regular well-developed pulsation sets in for higher gravity and irregular pulsation for lower gravity (see, Fig. 11).

It is very impressive that the variation amplitudes due to the instability of high-order modes are quite small compared with those from the instability of low-order modes. The highorder modes that are responsible to the variation are strange modes, and they have pulsation periods close to those of strongly damped modes, so nonlinear coupling between the strange modes and the very strong damped modes should be strong. This may suppress the amplitude of high-order modes drastically.

It is interesting to see the transition from regular pulsation to irregular pulsation as seen in Figs. 7-11 at low values of $\log g$. The period doubling (Buchler et al. 1987) and the intermittency (Aikawa 1987) have been found in pulsation models as the transition routes from regular to irregular pulsation. To see such a transition in the present model sequence, more fine tuning of the model parameters would be necessary.

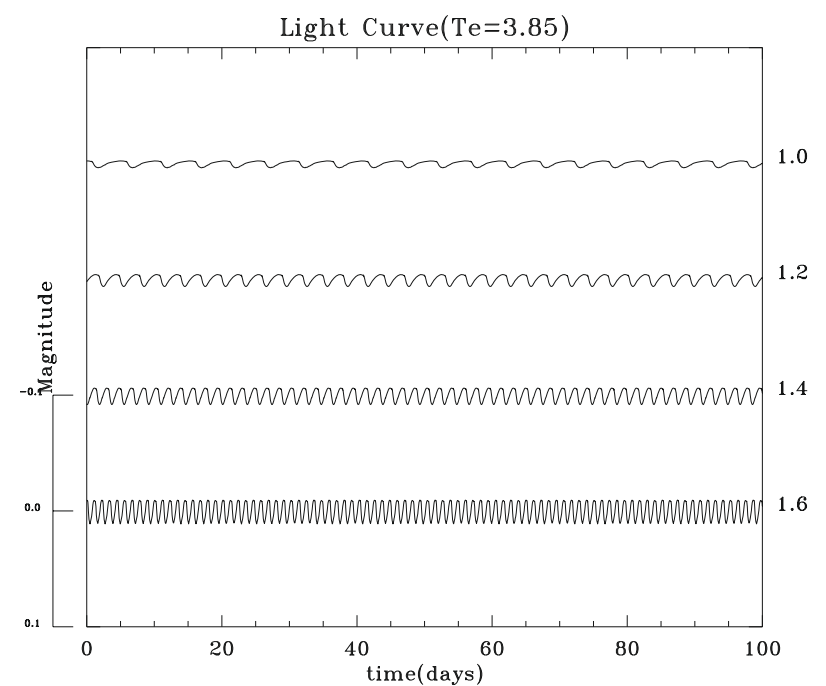

Fig. 9. Same as in Fig. 6, but for $\log T_{\text {eff }}=3.85$ of $M=0.8 M_{\odot}$.

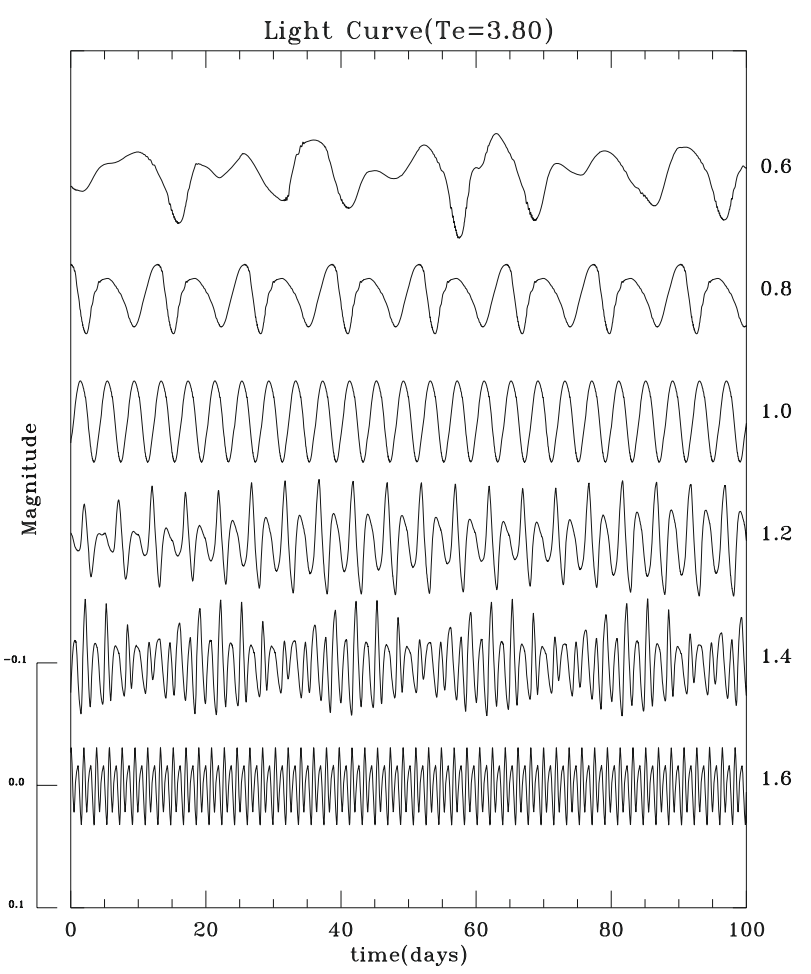

Fig. 10. Same as in Fig. 6, but for $\log T_{\text {eff }}=3.80$ and $M=0.8 M_{\odot}$.

\section{Discussion and concluding remarks}

The instability strip for post-AGB pulsation was examined. On the bluer side of the classical instability strip of low-order pulsation modes, there is a significant size of the instability domain of high-order modes. The region may be responsible for the photometric variability of some post-AGB stars with small peak-topeak variations.

For the models with the parameters of the bluer instability region, nonlinear simulations performed with a hydrodynamic code with the variable Eddington approximation of radiative transfer reveal small peak-to-peak variations like those observed in some of post-AGB stars. The time scale of the variations in general is comparatively shorter than that observed (for instance, Hrivnaket al. 2001). However, Bond et al. (1984) describe the variation of HD 46703 (which is included in Fig. 1) as brightness 


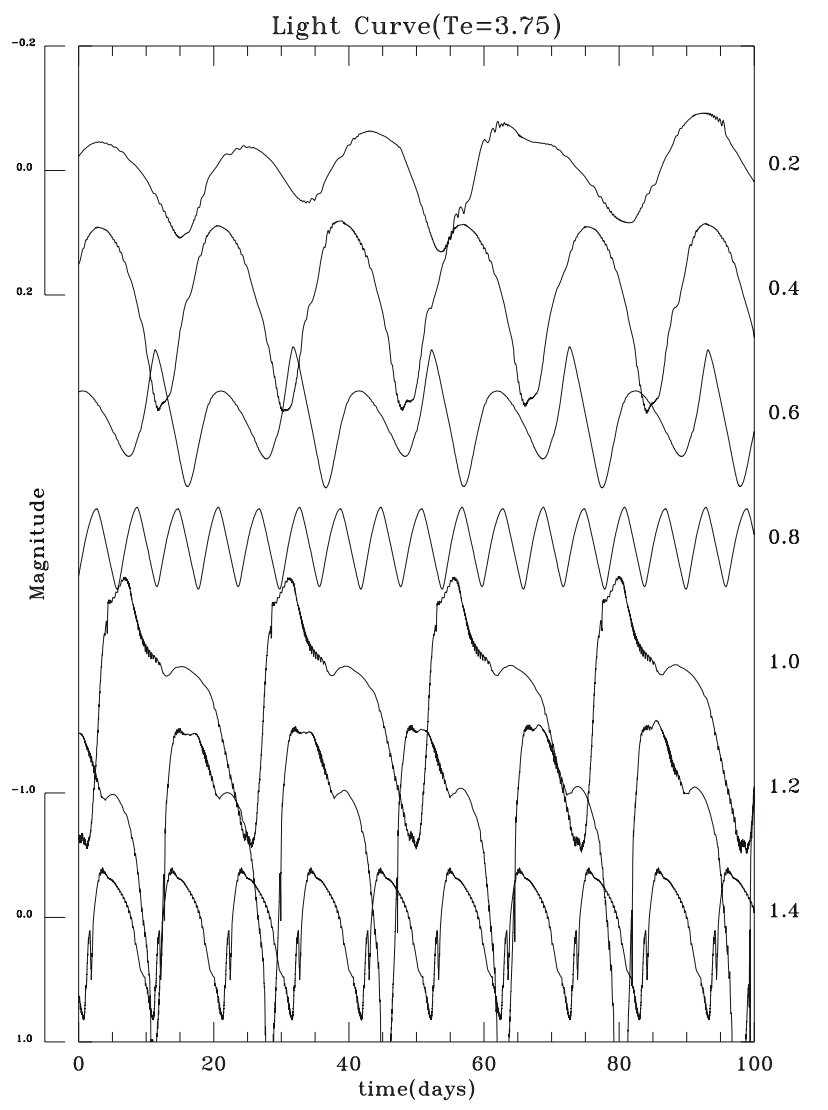

Fig. 11. Same as in Fig. 6, but for $\log T_{\text {eff }}=3.75$ and $M=0.8 M_{\odot}$. The upper scale is applied to the upper four curves, and the lower scale is valid for the lower three curves.

variations of $0.1 \mathrm{mag}$ on the time scale of a week. The pulsation behavior of our models may be a perfect match to this star.

To get the stationary pulsation state of nonlinear models, we met some difficulties on numerical treatments during the nonlinear simulation. Starting with the initial conditions as mentioned before, we could run the models for a while, but the convergence of implicit calculation became very poor, and so we could not continue the simulation to confirm stationary state of nonlinear pulsation. Those are models with higher $\log T_{\text {eff }}$, $\operatorname{lower} \log g$ (for instance, $\log T_{\text {eff }}=3.80, \log g=0.2$ ), and $\operatorname{lower} \log T_{\text {eff }}$ (for instance $\log T_{\text {eff }}=3.70$ ). The first may be related to the opacity-modified Eddington limit (Asplund 1998). The region where the opacity-modified Eddington limit realized so the radiation pressure is dominant becomes close to the dynamical instability. Tuchman et al. (1979) show that pulsation becomes violent and mass loss may occur in the model envelope close to the dynamical instability. We confirmed that the velocity of the outer shell reached the escape velocity during pulsation. We need a pulsation code that includes running waves for these stars. The second is the models in the redder region of the instability strip and nonlinear amplitudes became too large to continue the simulation, so our analysis should include some effects of convection and/or pulsation-induced turbulence to get the stationary state of moderate nonlinear amplitudes. There have been some attempts as nonlinear pulsation related to the region (Fadeyev \& Tutukov 1981; Fadeyev 1982; Fadeyev \& Muthsam 1990).

RV Tau stars are mainly located in the main part of the instability strip of post-AGB stars. Pulsation behavior of the models for parameters corresponding to that region should reproduce the photometric variations of RV Tau stars that are supposed to be post-AGB stars. AC Her and R Sct are ones that have been observed well and monitored for a long time. Kolláth (1990) and Kolláth et al. (1998) compiled data of the photometric variation of these two stars and tried to characterize the long-monitored pulsation. At the same time, they mention that the behavior of the variations of these two stars is quite different. The difference may come from the their locations located at different parts of the instability strip, because the effective temperatures of R Sct is estimated as $4500 \mathrm{~K}$, while it is $5900 \mathrm{~K}$ for AC Her. This difference may cause the quite large difference in photometric variation, because AC Her may be located at the edge of the instability bay with a small amplitude pulsation, while R Sct is located well within the main instability strip. It is interesting to compare the models with observations using these well monitored data. We will discuss this problem in a future paper.

Acknowledgements. Part of this work was supported by the Japanese Grantin-Aid for Scientific Research of the Ministry of Education, Culture, Sports, Science, and Technology, project number 14540229.

\section{References}

Aikawa, T. 1987, Ap\&SS, 139, 115

Aikawa, T. 1991, ApJ, 374, 700

Aikawa, T. 1993, MNRAS, 262, 893

Aikawa, T. 2008, A\&A, 484, 419

Aikawa, T., \& Screenivasan, R. 1996, Pub. Astron. Soc. Japan, 48, 29

Allen's Astrophysical Quantities (Fourth Edition), Cox, A. N. (ed.), 2000, AIP Press

Alexander, D. R., Ferguson, J. W. 1994, ApJ, 437, 879

Asplund, M. 1998, A\&A, 330, 641

Barthes, D., Lebre, A., Gillet, D., \& Mauron, N. 2000, A\&A, 359, 168

Blöcker, T. 1995, A\&A, 299, 755

Bond, H. E., Carney, B. W., \& Grauer A. D. 1994, PASP, 96, 176

Bridger, A. 1985, in Cepheids: Theory and Observations, ed. Madore B. (CUP), 246

Buchler, J. R., Goupil, M.-J., \& Kovacs, G. 1987, Phys. Lett. As, 126, 177

Castor, J. I., Davis, C. G., \& Davison, D. K. 1977, Los Alamos Scientific Lab., LA-6664

Córsico, A. H., Althaus, L. G., Miller Bertolami, M. M., \& Werner, K. 2007, A\&A, 461, 1095

Davis, C. D. 1972, ApJ, 172, 419

Fadeyev, Yu. A. 1982, Ap\&SS, 86, 143

Fadeyev, Yu. A., \& Lynas-Gray, A. E. 1996, MNRAS, 209, 38

Fadeyev, Yu. A., \& Muthsam, H. 1990, A\&A, 234, 188

Fadeyev, Yu. A., \& Tutukov, A. V. 1981, MNRAS, 195, 811

Frankowski, A. 2003, A\&A, 265, 271

Hrivnak, B. J. et al. 2001, in Post-AGB Objects as a Phase of Stellar Evolution, ed. Szczeba R., \& Górn, (Kluwer AP), 101

Jeannin, L., Fokin, A. B., Gillet, D., \& Baraffe, I. 1996, A\&A, 314, L1

Jeannin, L., Fokin, A. B., Gillet, D., \& Baraffe, I. 1997, A\&A, 326, 203

Jeffery, C. S., \& Schönberner, D. 2006, A\&A, 459, 885

Kiss, L. L., Derekas, A., Szabó, M., Bedding, T. R., \& Szabados, L. 2007, A\&A, 375,1338

Kolláth, Z. 1990, MNRAS, 249, 377

Kolláth, Z., Buchler, J. R., Serre, T., \& Mattei, J. 1998, A\&A, 329, 147

Paczyǹski, B. 1971, AcA, 21, 417

Saio, H. 1995, MNRAS, 277, 1393

Saio, H., \& Jeffery, C. S. 1988, ApJ, 328, 714

Saio, H., Wheeler, J. C., \& Cox, J. P. 1984, ApJ, 281, 318

Schönberner, D. 1981, A\&A, 103, 119

Schönberner, D. 1983, A\&A, 272, 708

Schmidt, M. R. 2001, in Post-AGB Objects as s Phase of Stellar Evolution, ed. Szczeba R., \& Górn, (Kluwer AP), 271

Seaton, M. J. 1992, MNRAS, 265, 125

Seaton, M. J., Yan, Yu., Mihalas, D., \& Peadhan, A. K. 1994, MNRAS, 266, 805 Stellingwerf, R. F. 1975a, ApJ, 195, 135

Stellingwerf, R. F. 1975b, ApJ, 199, 705

Stothers, R. B. 2003, ApJ, 589, 960

Szczerba, R., Siòdmiak, N., Stasiǹska, G., \& Borkowski, J. 2007, A\&A, 367, 799 (http: //www.ncac. torun.pl/postagb)

Tuchman, Y., Sack, N., \& Z. Barkat 1979, ApJ, 234, 217

Whitney, C. A. 1978, ApJ, 220, 245

Wood, P. R. 1976, MNRAS, 174, 531

Zalewski, I. 1992, PASJ, 44, 27 